\title{
Plasmonic properties and energy flow in rounded hexahedral and octahedral nanoparticles
}

\author{
Dimitrios C. TZarouchis ${ }^{1, *}$, Pasi Ylä-OIJala ${ }^{1}$, Tapio Ala-Nissila ${ }^{2,3}$, And Ari Sihvola ${ }^{1}$ \\ ${ }^{1}$ Department of Radio Science and Engineering, Aalto University, P.O. Box 13000, FI-00076 Aalto, Finland \\ ${ }^{2}$ COMP Centre of Excellence, Department of Applied Physics, Aalto University, P.O. Box 111000, Fl-00076 Aalto, Finland \\ ${ }^{3}$ Department of Physics, Brown University, P.O. Box 1843, Providence, Rhode Island 02912, USA \\ *Corresponding author: dimitrios.tzarouchis@aalto.fi
}

Compiled September 12, 2016

\begin{abstract}
The resonant plasmonic properties of small dual rounded nano-scatterers are numerically investigated. A set of Drude-like, silver hexahedral and octahedral structures are studied and compared with a reference spherical particle through a numerical surface integral equation technique. Surface, near field, Poynting field and streamline distributions are presented illustrating novel plasmonic features arising from the complex shapes of the nanoparticles, while several designing rules are described. These qualitative observations can be used appropriately towards sensing, sorting, harvesting, and radiation control applications. () 2016 Optical Society of America
\end{abstract}

OCIS codes: 290.0290 Scattering; 290.5850 Scattering, particles; 250.5403 Plasmonics; 200.4690 Morphological transformations; 160.4760 Optical properties; 050.1755 Computational electromagnetic methods.

http://dx.doi.org/10.1364/ao.XX.XXXXXX

\section{INTRODUCTION}

Radiative scattering properties of nanoparticles/scatterers (NPs) in visible and infrared wavelengths (vis-IR) comprise an intensively studied, multidisciplinary topic with a plethora of applications in the realm of nanotechnologies [1]. At this range, NPs made from materials such as silver, aluminum, or gold exhibit collective electron oscillations, known as plasmonic or localized surface plasmon resonances (LSPR) [2]. These highly tunable resonances place NPs in the center for both passive [3, 4], and active [5] optical control applications. Almost in every case the spectral behavior and the main scattering mechanisms are affected by both the material and the geometry of the inclusions. Hence, understanding these effects of more complex geometries is of great importance, especially for novel ideas on controlling the vis-IR radiation.

A qualitative, yet fundamental perspective on exploring scattering mechanisms of isolated NPs has been given independently by Bohren [6] and Paul [7], where a Poynting flow line perspective of the scattering mechanism of a sphere has been presented. These works have delivered insights regarding the scattering/absorption mechanisms, especially in the vicinity of small resonant spheres. Conceptually, the electromagnetic energy flow resembles the hydrodynamic flow [8, 9], demonstrating how the structure perturbs its near field distribution in a scattering problem setup. From a mathematical point of view, the perturbed near field distribution may exhibit several critical points, such as centers and saddles, whose behavior is affected by the scatterers' geometry $[8,9]$.

Recent studies shed new light on the scattering mechanisms by small nano-spheres/spheroids and their energy flow at the plasmonic resonance [10-12], where several critical points have been identified in the near field of the scatterers. These results elucidate the fact that the near field power distribution is highly affected both by the geometry and the material losses.

Later works have extended these studies to higher order plasmonic resonances [13], utilizing the same analytical methods, i.e., Mie scattering theory [14]. Analytical methods also have been used for analyzing other geometrical shapes, such as core-shell spheres [15] and circular cylinders [3, 16]. Interestingly, novel particle functionalities and designs could be devised [3, 17, 18] by exploiting the near field setup and the Poynting streamline distribution.

An alternative view on the near-field/Poynting distribution problem can be obtained by matching LSPRs to 
classical antenna theory; all scattering effects can readily be studied from an antenna point of view, as long as quantum effects do not need to be explicitly taken into account. This fact allows us to exploit almost every antenna design tool available for device engineered purposes, especially on understanding how the morphology affects the overall resonant behavior. For instance in [5], a cube-like NP is utilized as a patch nano-antenna providing a large emission rate enhancement. The key point on this analysis is the fact that cube exhibits enhanced, corner induced field distribution on its dipolar plasmonic resonance, qualitatively described as a microstrip patch antenna.

In the present work the spectral behavior and near field properties of regular rounded hexahedral (cuboid) and octahedral (lozenge) shapes are studied through a surface integral equation (SIE) numerical scheme [19]. These rounded closed structures belong to the set of superquadric [20] surfaces, generalizing is a sense the family of regular Platonic solids [21]. Superquadric shapes with rounded corners, such as cubes (regular hexahedra) and lozenges (regular octahedra), are of particular interest either as realistic deformations of spherical particles and clusters, or as finely engineered nano-antennas and NPs. Therefore, studying these shapes expands our understanding towards realistic NPs and their implementation into novel designs and applications.
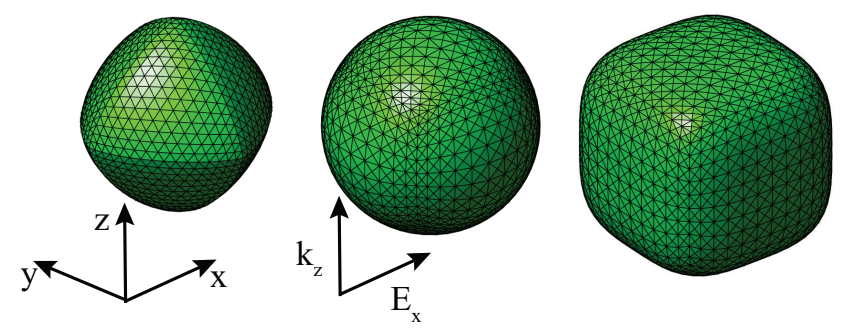

Fig. 1. Mesh view generated by Eq. (2) for the nanoparticles studied here. Left: lozenge of $p=0.7$. Center: sphere of $p=1$. Right: cuboid of $p=1.75$. All shapes are described by the same scaling factor $d=25 \mathrm{~nm}$. The external field is $x$ polarized and propagates in the $z$ direction.

\section{THEORY}

\section{A. Surface Integral Equation Method}

The field distributions and scattering cross sections of plasmonic nano-particles are computed with the surface integral equation (SIE) method. This widely used method offers accurate and reliable solutions for many computational electromagnetics problems [22], and has been recently extended [23] for modeling plasmonic scatterers and nanoantennas [24-27].

The SIE method is based on Love's equivalence principle, allowing the reformulation of the original problem of solving Maxwell's equations in the entire 3D space as an equivalent problem of solving equivalent sources (currents) on the surfaces and interfaces. The method is thus especially well suited for modeling LSPRs where important physical phenomena take place on the surfaces.

The method used here is described in detail in [19], and the singular integrals involved are evaluated with the singularity subtraction technique available in $[28,29]$. Accurate evaluation of these integrals is important to maintain the accuracy of the solution, particularly in the near field region. The scattered and absorbed power can be efficiently evaluated using the associated SIE matrices as explained in [30].

\section{B. Remarks on superquadric shapes}

The systematic implementation of the SIE method to an arbitrarily shaped scatterer requires the proper discretization of the particle's surface [19]. Often, a mathematically convenient way to describe a given surface is by using its parametric description.

Here we focus mainly on three special cases of the superquadric surfaces, i.e., a sphere, a rounded hexahedron (cuboid), and a rounded octahedron (lozenge). The aforementioned shapes are parametrically represented as a set of sinusoidal equations [20], viz.,

$$
r(\theta, \phi)=\left(\begin{array}{c}
a_{1}|\sin \vartheta \cos \varphi|^{1 / p} \\
a_{2}|\sin \vartheta \sin \phi|^{1 / p} \\
a_{3}|\cos \vartheta|^{1 / p}
\end{array}\right),
$$

where $0 \leq \vartheta \leq \pi$ and $0 \leq \varphi \leq 2 \pi$ are the spherical coordinate system variables. The $a_{1}, a_{2}$, and $a_{3}$ terms are the axial scaling factors, and $p$ is the superquadric rounding/squareness factor $\left(\frac{1}{p}\right.$ also known as roundness [20]). In our case the scaling factors are equal to $a_{1}=a_{2}=a_{3}=d / 2$, where $d$ is sphere's diameter. Note that Eq. (1) can be easily transformed to its inside-out equivalent function [20]

$$
f(x, y, z)=\left|\frac{x}{a_{1}}\right|^{2 p}+\left|\frac{y}{a_{2}}\right|^{2 p}+\left|\frac{z}{a_{3}}\right|^{2 p},
$$

where $x, y$, and $z$ are the Cartesian coordinates.

As an example, when $p=1$ Eq. (2) describes a sphere of radius $\frac{d}{2}$ (Fig. 1 center). Squareness values above unity $(p>1)$ result in a superquadric cube with rounded corners (cuboid, Fig. 1 right), leading to a perfect cube for $p \rightarrow \infty$. For values between $\frac{1}{2}<p<1$ a rounded lozenge shaped surface is formed (Fig. 1 left). Finally, for smaller values $\left(p<\frac{1}{2}\right)$, pinched superquadric surfaces occur. In our analysis we will study structures within $p \in\left[\frac{1}{2}, \infty\right)$ range.

Another interesting property of these surfaces is that the normal vector with respect to surface expressed in Eq. (1) can be described as a parametric expression by mutually substituting the corresponding scaling factors with the inverse $\frac{1}{a_{i}}(i=1,2,3)$, and the rounding factor by 

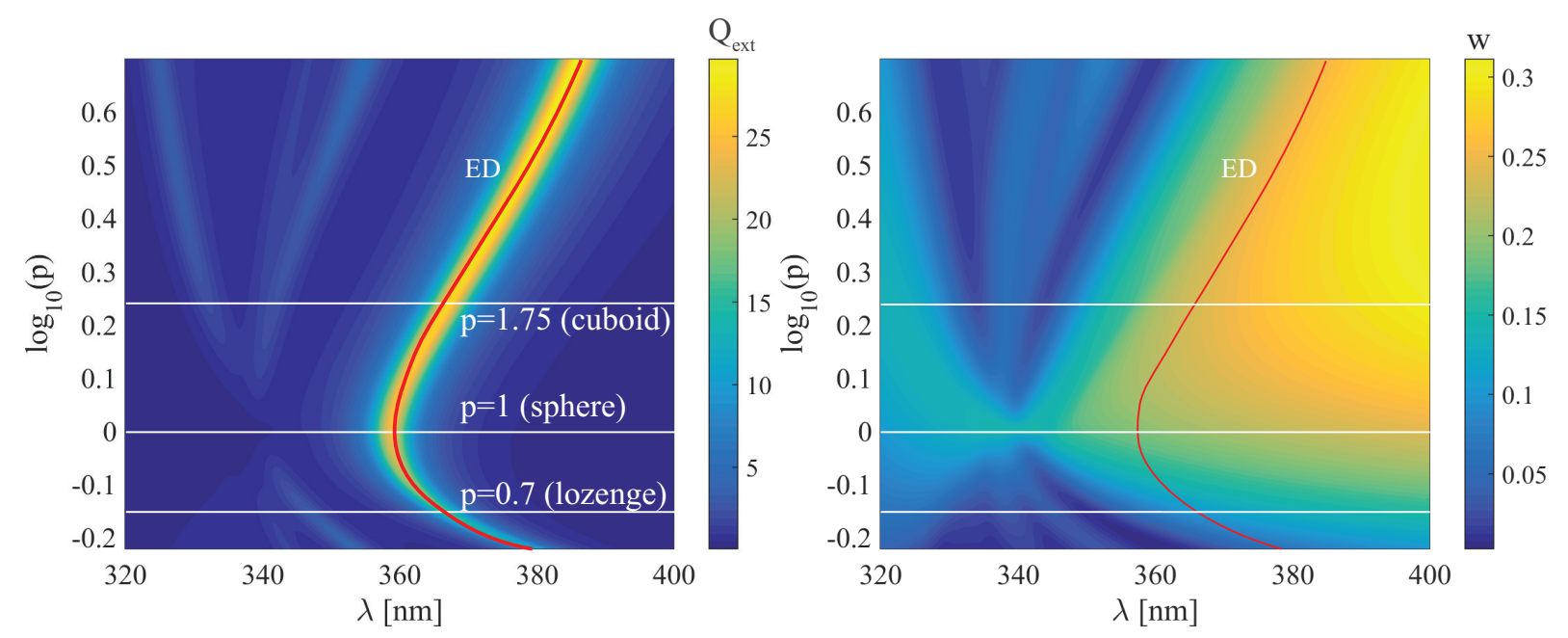

Fig. 2. Shape transformation mappings as a function of the rounding factor $p$ for silver NPs. Left: the extinction efficiency (ratio of the extinction cross section over the geometrical cross section, i.e., $Q_{e x t}=\sigma_{e x t} / C_{g}$ ) in the range $0.6 \leq p \leq 5$. The electrical dipole-like (ED) resonance is visible (red line). Right: the plasmonic albedo, i.e., the ratio between the scattered over the extinction cross sections $\left(w=\sigma_{s c a} / \sigma_{\text {ext }}\right)$ [31]. Notice that the ED resonance exhibits a smooth albedo transition, while lower albedo values for the higher modes are manifested.

the following expression

$$
p_{d}=\frac{p}{2 p-1}
$$

The surface obtained is characterized as dual to the original one [20] obtained by Eq. (1). Interestingly, this observation gives a direct connection between cuboid and lozenge-like shapes, based to the above fact, e.g., a cuboid of $p=1.75$ (Fig. 1 right) corresponds to a dual lozenge surface of $p_{d}=0.7$ (Fig. 1 left). For the case of a perfect hexahedron $(p \rightarrow \infty)$ and octahedron $\left(p=\frac{1}{2}\right)$ the duality is expressed by the Euler characteristic rule for the Platonic polyhedra: Vertices + Faces $=$ Edges +2 [32]. According to this rule an octahedron is dual to a hexahedron by mutually substituting their number of vertices with the number of edges.

Last, the required cross sectional area, $C_{g}$, and volume, $V$ of each NP for the simple case of $a_{1}=a_{2}=a_{3}=d / 2$ is analytically obtained from the following formulas [33]

$$
\begin{gathered}
C_{g}=\frac{d^{2}}{2 p} B\left(\frac{1}{2 p}, \frac{1}{2 p}+1\right) ; \\
V=\frac{d^{3}}{4 p^{2}} B\left(\frac{1}{p}, \frac{1}{2 p}+1\right) B\left(\frac{1}{2 p}, \frac{1}{2 p}\right),
\end{gathered}
$$

where $B$ is the beta function [34]. Note that the volume of a sphere is $V_{s}=\frac{\pi}{6} d^{3}$. For perfect polyhedra the volume of a hexahedron is $V_{c}=d^{3}$, while an octahedron occupies one sixth of the volume, i.e., $V_{l}=\frac{d^{3}}{6}$.

\section{RESULTS \& DISCUSSION}

\section{A. Extinction, absorption, and single plasmonic albedo}

Let us begin by introducing the reference NP, a silver sphere of diameter $d_{\text {sphere }}=25 \mathrm{~nm}$ (Fig. 1 center). Silver is a widely used reference material, mostly due to its strong plasmonic resonances occurring naturally at the opticalnear ultraviolet spectrum [2]. The material parameters used follow a simple Drude-like dispersion model,

$$
\epsilon_{A g}(\lambda)=\epsilon_{\infty}+\frac{\left(\lambda / \lambda_{p}\right)^{2}}{1-j \lambda / \lambda_{d}}
$$

where $\epsilon_{\infty}=5.5, \lambda_{p}=130 \mathrm{~nm}$, and $\lambda_{d}=30 \mu \mathrm{m}$, introduced in [35]. This model is a curve fit on Johnson's and Christy's [36] experimental data for bulk silver.

Despite the fact that the optical response of small particles is expected to be slightly different from the bulk limit [37], our analysis concentrates in exploring and comparing the general behavior of the main plasmonic resonances resulting in a commonly modeled Drude-like medium reference, i.e., simple Ag-Drude model experiencing low-to-moderate level of dissipative losses. In that sense the analysis is readily expanded for any natural or artificial dispersive material following the simple Drude model.

It is well known that spherical Ag particles exhibit pronounced plasmonic resonances approximately at $\lambda=$ $360 \mathrm{~nm}$, i.e., size parameter $x \approx 0.2$. This LSPR is described by an electric, dipole-like behavior [2]. Note that a $z$ propagated, $x$ polarized field is assumed throughout the whole study (see Fig. 1).

Since the geometries studied can be defined by the same set of parametric equations, unified transformation mappings can be easily extracted, revealing the extinction efficiency spectrum as a function of both the wavelength $\lambda$ and the squareness factor $p$ (Fig. 2). Moving away from the sphere $p=1$ the resonant spectrum becomes richer and the main dipole resonance is red-shifted. These higher order resonances verify already known re- 
sults, see e.g. Fuchs [38], that more than one pronounced resonance may appear for the case of small cubic particles. Here, in Fig. 2 these results are further extended for the case of lozenge particles where a vibrant resonance spectrum is obtained. Indeed, corners are able to induce distinctive plasmonic resonances [39], even for the case of subwavelength particles.

An alternative way to quantify the triggered scattering mechanisms is by plotting the single particle plasmonic albedo, as illustrated in Fig. 2. Albedo is defined as the ratio between the scattered over the extincted power [31]; a qualitative figure exposing the balance between the absorption and scattering mechanisms [40]. Here the maximum albedo level is $\approx 0.3$, a value indicating absorption being the main extinction mechanism in all cases. This is a common feature for all subwavelength NPs where extinction is mainly attributed to materially induced absorption while scattering is almost three orders of magnitude smaller [31].

Another interesting feature occurs by comparing both panels in Fig. 2, where the main resonance has disappeared from the albedo map. The main dipole-like resonance exhibits a smoother albedo for all the shapes in the transformation mapping (Fig. 2, right panel). This result points to the fact that the absorptive characteristics of a subwavelength particle can be geometrically enhanced especially for the higher order resonances, i.e., appearance of valleys in Fig. 2 in both the cuboid $\left(\log _{10}(p)>0\right)$ and the lozenge $\left(\log _{10}(p)<0\right)$ regions. Lower albedo values are observed at the lozenge region, with respect to the cuboid. This is mainly attributed to the overall volume difference between the cuboid and lozenge.

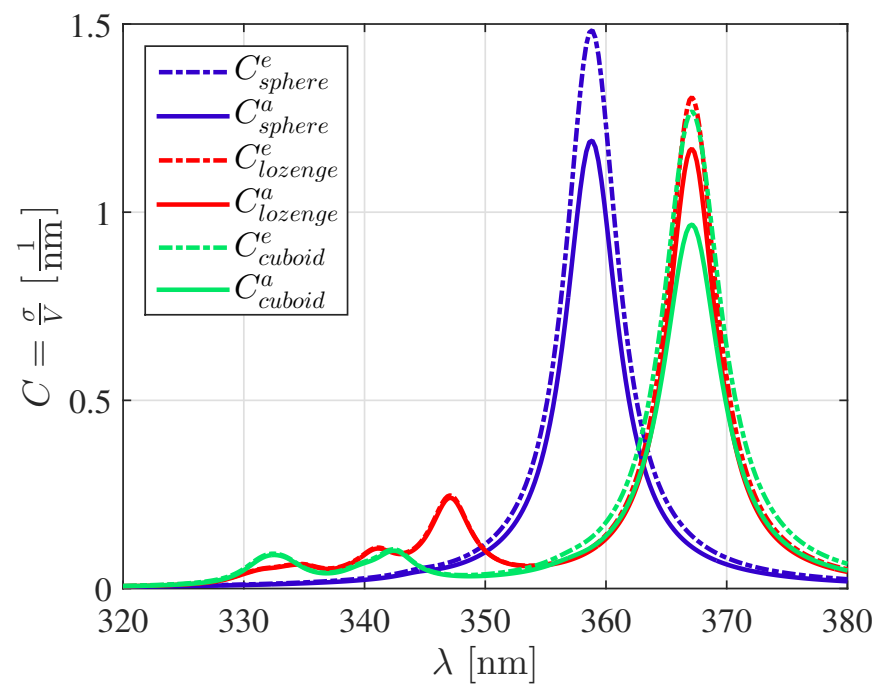

Fig. 3. Volume-normalized extinction, $C^{e}=\sigma_{\text {ext }} / V$ (dashed lines), and absorption, $C^{a}=\sigma_{\text {ext }} / V$ (solid lines), for the case of a silver sphere (blue, $p=1$ ), a cuboid (green, $p=1.75$ ), and a lozenge (red, $p=0.7$ ) NPs. Utilizing Eq. (5), the volume ratio between the cuboid and lozenge is approximately $V_{c} / V_{l}=2.32$.

\section{B. Dual shapes and plasmonic resonances}

Smoothly cornered cuboid structures can be perceived as perturbed spheres exhibiting dipole resonance red-shift with enhanced higher multipole spectrum [40,41]. This fact is generalized here also for the the octahedron case, where a similar trends are observed.

Since these dual shapes can be conveniently described by the same parametric expression through the rounding factor $p$, a point of novel interest would be the connection of their resonant behavior based on this parameter. Studies on polarizable NPs possessing sharp corners reveal a wealth of resonances, attributed to their corners [2, 38, 4042]. Some of these corner induced resonant properties have been studied also through an eigenvalue perspective for the 2D case [39].

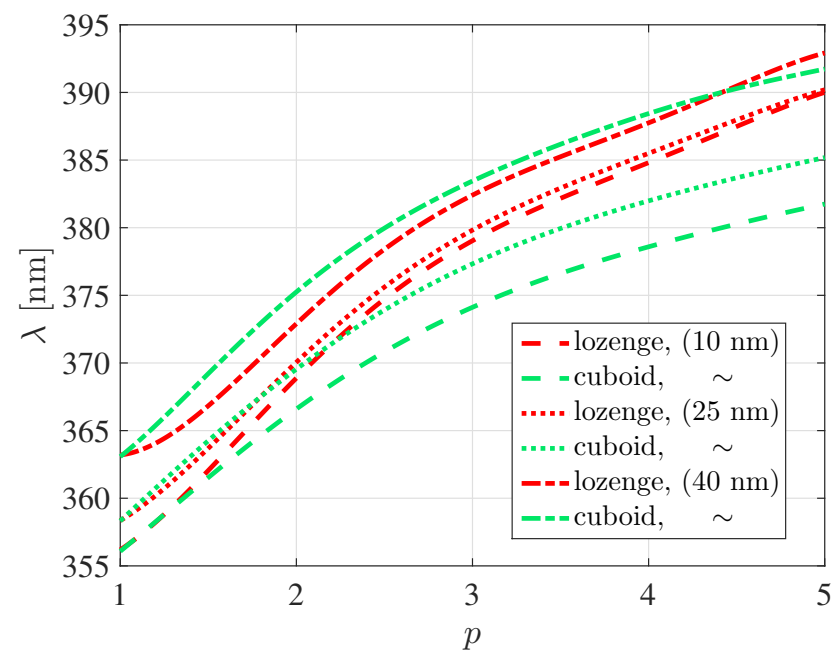

Fig. 4. Comparison of the position of the dipole plasmonic resonances as a function of the rounding factor $p$ : Green lines depict the resonant position of a $d=10 \mathrm{~nm}$ (dotted line), $d=25 \mathrm{~nm}$ (dashed line), and $d=40 \mathrm{~nm}$ (dotted-dashed line) cuboid, respectively. The red lines depict the lozenge resonant values for each size. Note that both cube and lozenge are commonly described through the duality formula found in Eq. (3).

The main dipole resonance of Fig. 2 (left) reveals that there is a value of $p$ where both the cuboid and lozenge resonate at the same wavelength. Indeed, Fig. 3 depicts the extinction and absorption volume normalized efficiencies for the $d=25 \mathrm{~nm}$ case, where both dual shapes ( $\left.p=1.75, p_{d}=0.7\right)$ resonate at the same wavelength. Additionally, their normalized extinction is almost at the same level, while there is an observed absorption difference. This difference is also visible in the albedo diagram (Fig. 2 right) where the albedo level is slightly smaller for the lozenge case and hence its absorption is enhanced. An intuitive explanation is that both particles' vertices and volume contribute to the overall scattering amplitude and the absorptive characteristics.

The picture above gives a hint whether this is true for the whole range of $p$ values, i.e., that the dual shapes 
resonate at the same frequency. Further analysis reveals that this trend is not accurately followed either for smaller or larger NPs, as can be seen in Fig. 4. For the case of a smaller scatterer $(d=10 \mathrm{~nm})$, the position of the resonant dipole diverges with increasing $p$, allowing only a small region with similar resonant wavelengths, i.e., up to $p=$ 1.5. For the $d=25 \mathrm{~nm}$ case the region is extended for values up to $p=2$. However, the largest NPs $(d=40 \mathrm{~nm})$ do not exhibit any similar effect except for a resonant crossing around $p=4.25$.

For all cases in Fig. 4 the lozenge resonates for longer wavelengths than the cuboid, for increasing values of $p$. Since all the particles studied can be characterized as subwavelength (size $\approx \lambda / 10$ ), the above observation agrees with the predicted electrostatic values for perfect Platonic solids, where the octahedra exhibit their first plasmonic resonance at larger wavelengths in comparison to the hexahedra (see the Appendix for details).

The rounding effect, combined with the albedo information (Fig. 2) could be potentially used as a guideline for designing NPs with enhanced absorption characteristics (lozenge). Again, the volume ratio between the cuboid and lozenge can be up to 6 times for the sharp cornered cases. In this sense lozenge shaped NPs might be suitable candidates for applications where enhanced absorption with less material volume is required.

\section{Surface fields and Poynting flowlines}

The extinction and albedo characteristics describe a smooth resonant transition with respect to the rounding factor for the main dipole resonance. Similar qualitative pictures can be extracted also by the surface charge and current distributions, illustrated in Fig. 5. Indeed, all scatterers exhibit similar dipole-like charge distributions, displaying small differences on the charge confinement amplitude, seen in Fig. 5 (a.1)-(a.3).

Specifically, Fig. 5 (a.3) depicts four distinctive regions for the cuboid NP. This result demonstrates the corner induced effects on a cuboid and its surface charge distribution, verifying already know results found in [41]. Here we extend these results to the lozenge case for which the surface charge distribution closely resembles the dipolelike charge distribution of the sphere, illustrated in Fig. 5 (a.2).

Consider now the current distribution in Fig. 5 b.1-3. In this case the cuboid (Fig. 5 b.3) exhibits an enhanced current amplitude but with a wider low current region on its sides. Surprisingly, the lozenge charge setup resembles almost a double-stream distribution (Fig. 5 b.1), with an overall decrease of the current amplitude. Therefore, several differences can be identified even for the simplest case of the first dipole resonance between the particles studied. These differences may be expected to have a significant impact on the near field distribution. Indeed, Figs. 6 and 7 illustrate the time averaged Poynting field distribution (background colormap) normalized to the incident power $\left(P_{0}\right)$ for each case (in logarithmic scale). The time averaged

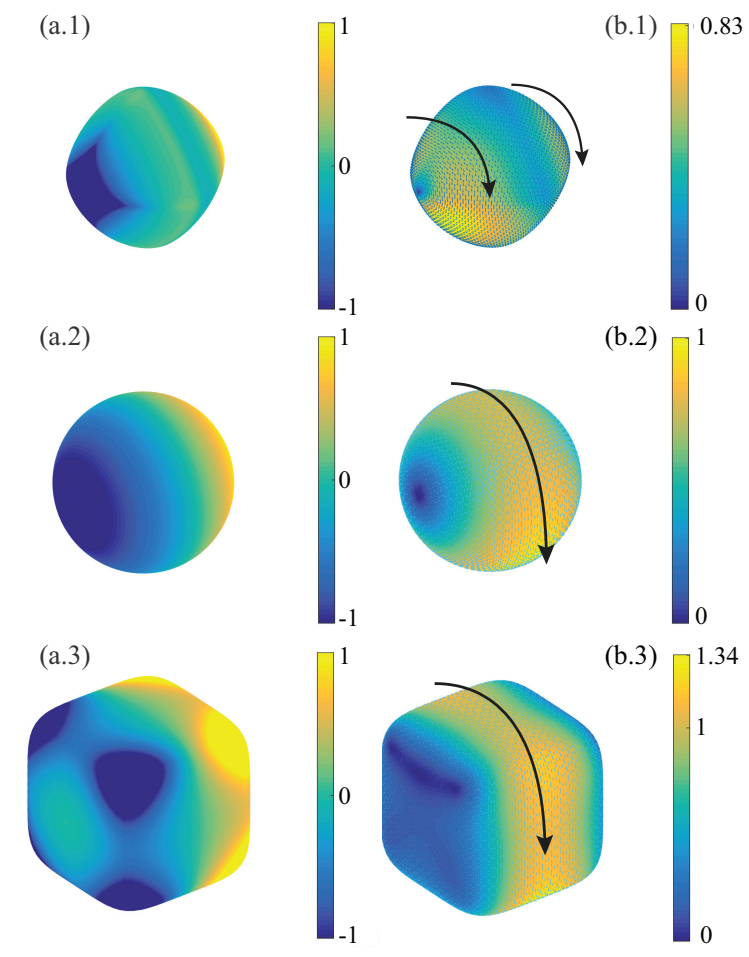

Fig. 5. The normal electric field (charges) for the first resonance of the $d=25 \mathrm{~nm}$ lozenge ( $p=0.7)$, sphere $(p=1)$, and cuboid ( $p=1.75)$ is depicted in (a.1)-(a.3), respectively. The values indicate the sign and charge confinement. The tangential magnetic field (currents) can be found similarly in (b.1)-(b.3). All values are normalized with respect to sphere's tangential field. Notice that the cuboid exhibits an enhanced circulating current, while the lozenge has two current paths of reduced intensity, with respect to the sphere.

Poynting field is defined as $\langle S\rangle_{t}=\langle\mathbf{E} \times \mathbf{H}\rangle_{t}$, assuming an $e^{-i \omega t}$ time dependence. It is important to notice that a similar Poynting "signature" is observed, for all NPs in the aforementioned figures. This fact justifies the characterization of the main resonance as a dipole also from the Poynting streamline perspective [43]. Likewise, each scatterer exhibits a similar near field power distribution lobe, while the minimum point (blue dot in Fig. 6 and 7) confirms the existence of the same saddle point (described also in [12] for the spherical case).

The color distinction between the streamlines quantifies the extinction efficiency area (or length) [8, 12], determining the effective cross sectional length for each NP, at each plane $(E$ and $H)$. In other words, the Poynting streamline perspective allows us to visualize and measure roughly the effective area of the scatterer $[6,7]$. By combining these two effective lengths a rough estimate on NPs effective area can be obtained. For instance, the cuboid has an increased extinction efficiency which is also visible by the width of the blue streamlines in both Figs. 6 and 7. Another interesting fact is that the $H$-plane effective length is slightly larger than that of the E-plane, 


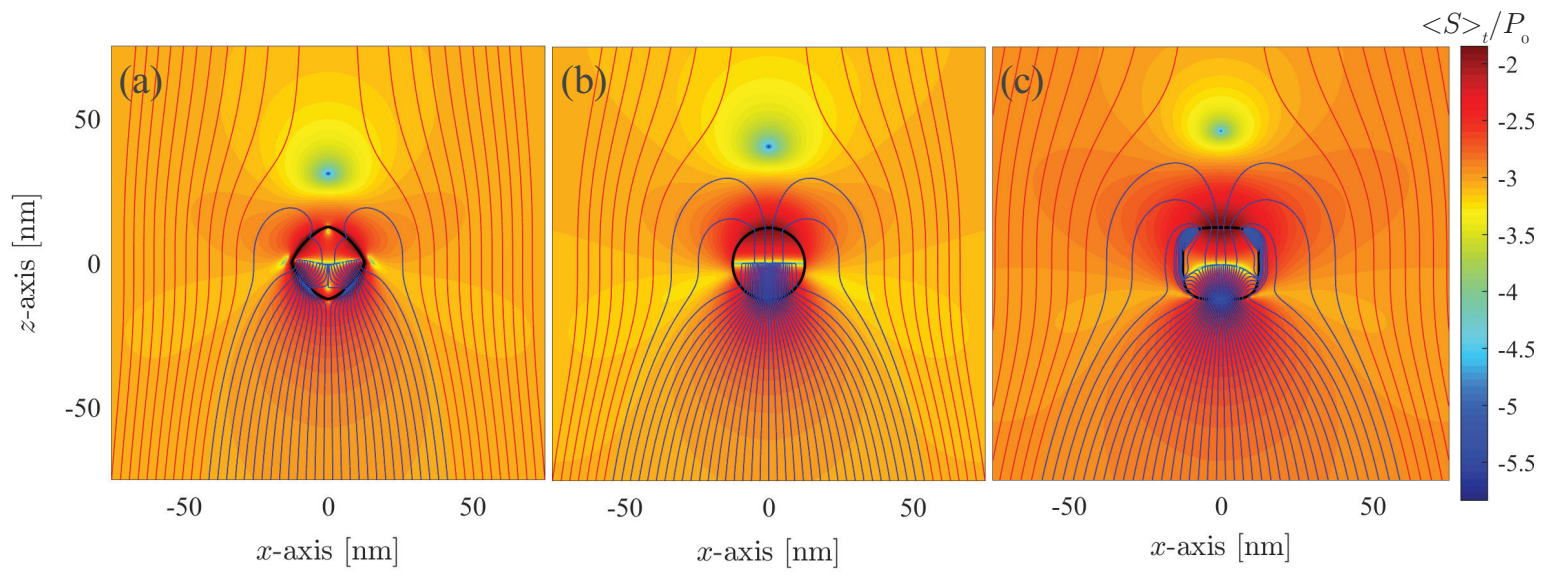

Fig. 6. Time-averaged Poynting amplitude (colormap) normalized with respect to the incident power in $\mathrm{dB}$ for (a) a lozenge of $p=0.7$, (b) a sphere of $p=1$, and (c) a cuboid of $p=1.75$, depicted in the the $E$-plane, assuming $z$ propagation and $E_{x}$ polarization. The red and blue lines illustrate the Poynting vector flow lines; blue-colored streamlines indicate the boundary of the effective extinction area.
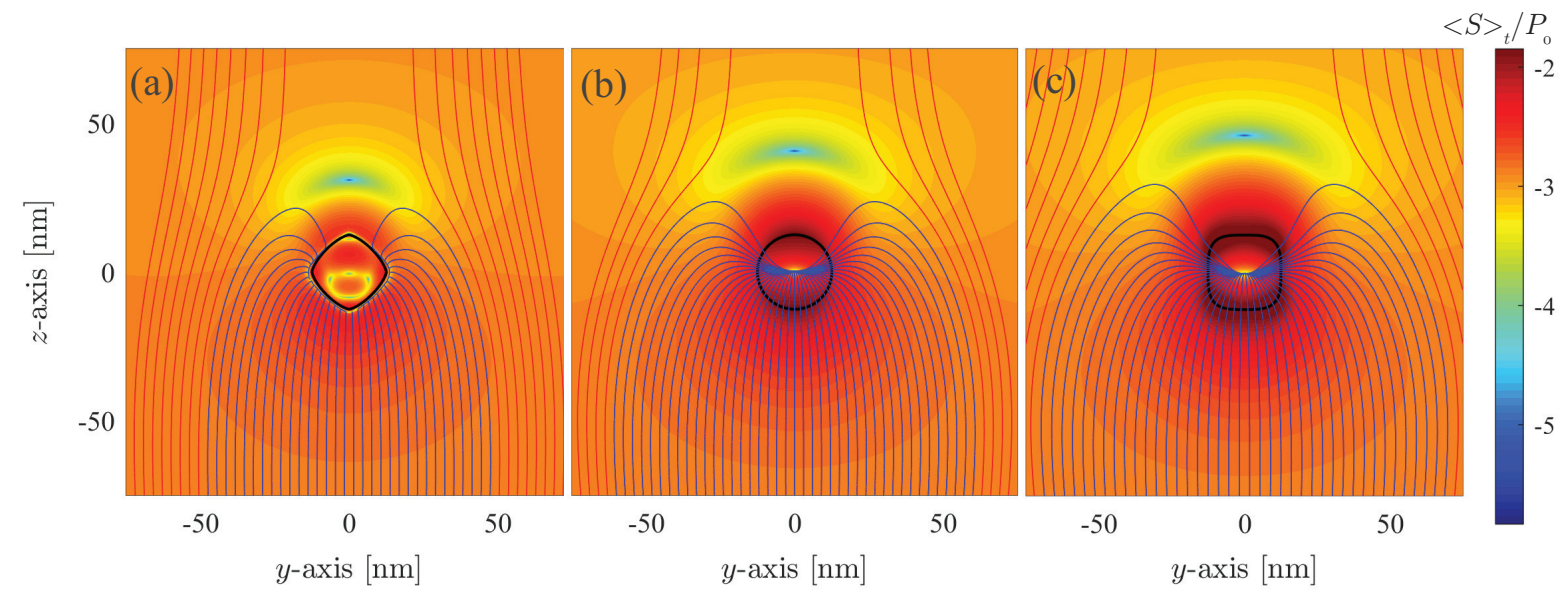

Fig. 7. Similarly to Fig. 6 the Poynting amplitude (colormap) in the the $H$-plane $\left(k_{z}, E_{x}\right)$. Notice different critical points and regions different from those in Fig. 6.

an indication that the cross sectional effective area is not symmetric. This observation can be connected with the effective area of a small dipole antenna where in general may have elliptical or even stranger shapes, as has been reported in [43].

Further analysis reveals more regions of interest in the cuboid and lozenge cases. To start with, the lozenge particle exhibits a smaller effective area, while new critical points appear inside and near its surface in both the $E$ and $H$-planes (Figs. 6 (a) and 7 (a)), respectively. One interesting aspect is that in the $\mathrm{H}$-plane, (Fig. 7 (a)) the streamlines are concentrated right on the surface of the particle. This observation might be of particular interest especially for applications that require part of the energy focused just on the particle surface.

The streamline pattern of the cuboid is equally interesting and rich. Here the effective area is increased, while new critical points appear near its corners, revealing two vortices where streamlines converge. Again, this kind of pattern might be of particular interest not only for energy harvesting applications but also for applications exploiting optical forces on single scatterers [18]. This can be understood through the fact that the Maxwell stress tensor contains a Poynting vector component [18]. Therefore, any change in the Poynting field distribution may change the tensor distribution and hence the forces acting on the scatterer.

Arguably, a point of particular interest is the accuracy of the method used. It is known that cubic and other shapes with corners suffer from solution convergence issues. For example, plasmonic resonances for very sharpcornered structures may cause numerical inaccuracies [42]. Here, the rounding values used are small in order to avoid any sharp corner induced inaccuracies. Note that recent studies indicate SIE to be an accurate method for simulating plasmonic phenomena for certain shapes, such as cuboids [44] with respect to other available methods such the discrete dipole approximation. 
The findings above make clear that the scattering properties of small nanoparticles may exhibit important and critical differences in their near field distribution. Therefore, how and when does the streamline distribution change, with respect to the rounding factor? A series of illustrations in Fig. 8 depict that changes in certain streamline trends start to appear for values above $p=1.2$ (Fig. 8 (b)), for the cuboid case. The lozenge case exhibits its first streamline perturbation for values below $p<0.9$, illustrated in Fig. 9 (b). In this case, new critical points appear, affecting the streamline distribution mostly in the $H$-plane. Sharper corners are able to create pronounced

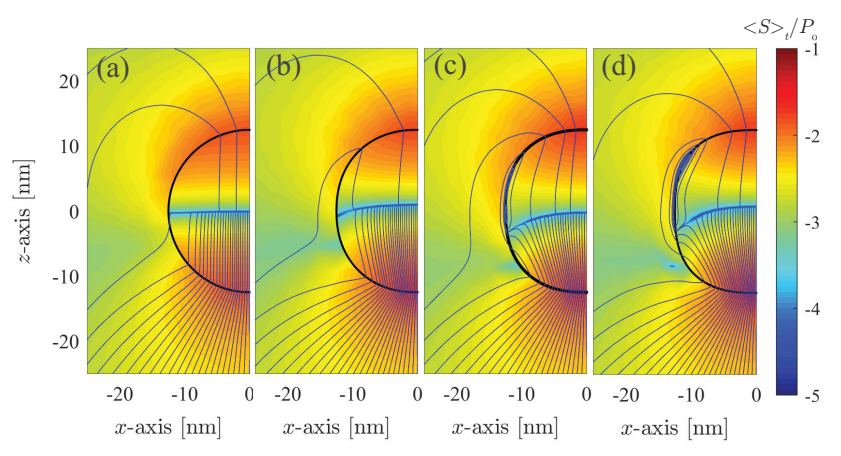

Fig. 8. Poynting streamline distribution in the E-plane for different rounding factors. (a) $p=1$, (b) $p=1.2$, (c) $p=1.4$, and (d) $p=1.6$. Notice that for values above $p=1.2$ new critical points (vortex and saddle) are gradually formed.

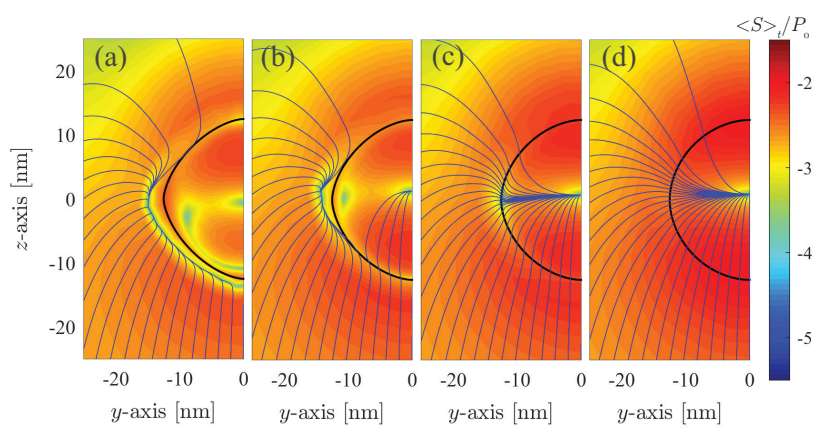

Fig. 9. Poynting streamline distribution in the $H$-plane for different rounding factors. (a) $p=0.8$, (b) $p=0.85$, (c) $p=0.9$, and (d) $p=0.95$. Notice that for values below $p=0.9$ several critical points gradually appear.

vortices in the Poynting streamline setup in both planes. This feature may be exploited for sensing, sorting, and measuring applications that take into account particles' roundness. Due to the fact that these differences appear in the Poynting field distribution, corners may facilitate the optical sorting of particles with many corners. Alternatively, cornered particles can be excellent candidates for biomedical or energy harvesting applications where enhanced power density is required close to the particles' surface.

\section{SUMMARY AND CONCLUSIONS}

In summary, several aspects on the plasmonic resonances of subwavelength, rounded nanoparticles with corners have been presented. Superquadric shapes, such as cuboids and lozenges, have been numerically studied generalizing the spherical NP case. Results on their extinction efficiency, surface and near field distributions have been obtained.

In particular, several qualitative results have been extracted, demonstrating corner effects in the scattering/absorption spectrum. The main dipole resonance exhibits smooth albedo level, featuring enhanced absorptive characteristics for all shapes. The Poynting streamline distribution reveals some geometrically induced peculiarities. Small shape perturbations lead to large streamline differences in both $E$ and $H$ planes. These qualitative observations can be further used as designing rules for synthesizing smooth or sharp cornered NPs, appropriately exploiting their functionalities for sensing, sorting, harvesting, and radiation control applications.

\section{ACKNOWLEDGEMENTS}

This work has been supported by the Aalto Energy Efficiency Programm, EXPECTS Project. D.T. would like also to acknowledge the ELEC Doctoral school funding scholarship for the support. T. A-N has been funded in part by the Academy of Finland through its COMP Center of Excellence program (grants no. 284621 and 287750).

\section{APPENDIX}

\section{Electrostatic polarizabilities of regular hexahedral and octahedral structures}

The dual regular hexahedron (cube) and octahedral (lozenge) structures are smooth versions of the Platonic solids [21]. Their electrostatic polarizabilities have been numerically extracted and a [4/4] Padé approximant has been calculated. The expression reads

$$
\alpha=\alpha_{\infty}(\tau-1) \frac{\tau^{3}+n_{2} \tau^{2}+n_{1} \tau-\alpha_{0}}{\tau^{4}+d_{3} \tau^{3}+d_{2} \tau^{2}+d_{1} \tau+\alpha_{\infty}}
$$

where $\tau=\varepsilon_{\text {inclusion }} / \varepsilon_{h}$ is the contrast permittivity, $\varepsilon_{A g}$ is the inclusion and $\varepsilon_{h}$ the host environment permittivity, respectively. The following table gives the coefficients for both the hexahedral and octahedral cases, with five digit accuracy.

Notice that a fourth order Padé expansion of the calculated polarizability gives four poles and thus four distinctive resonances for the extinction spectrum, depicted in Fig. 10. Moreover the main dipole resonance appears at higher frequencies for the octahedral case.

This trend matches the results of Fig. 4, where for large values of the rounding factor $p$ the octahedron resonates at slightly higher wavelengths than the hexahedron. As an example the first resonant value of octahedron is at $\varepsilon=-4.37$, while for the octahedron at $\varepsilon=-4.74$ 
Table 1. Padé coefficients of the polarizabilities

\begin{tabular}{ccc} 
& Hexahedron & Octahedron \\
\hline$\alpha_{0}$ & -1.6383 & -1.5871 \\
$\alpha_{\infty}$ & 3.6442 & 3.5507 \\
$n_{2}$ & 4.83981 & 5.13936 \\
$n_{1}$ & 5.54742 & 5.86506 \\
$d_{3}$ & 8.0341 & 8.26227 \\
$d_{2}$ & 19.3534 & 19.8267 \\
$d_{1}$ & 15.4349 & 15.6191
\end{tabular}

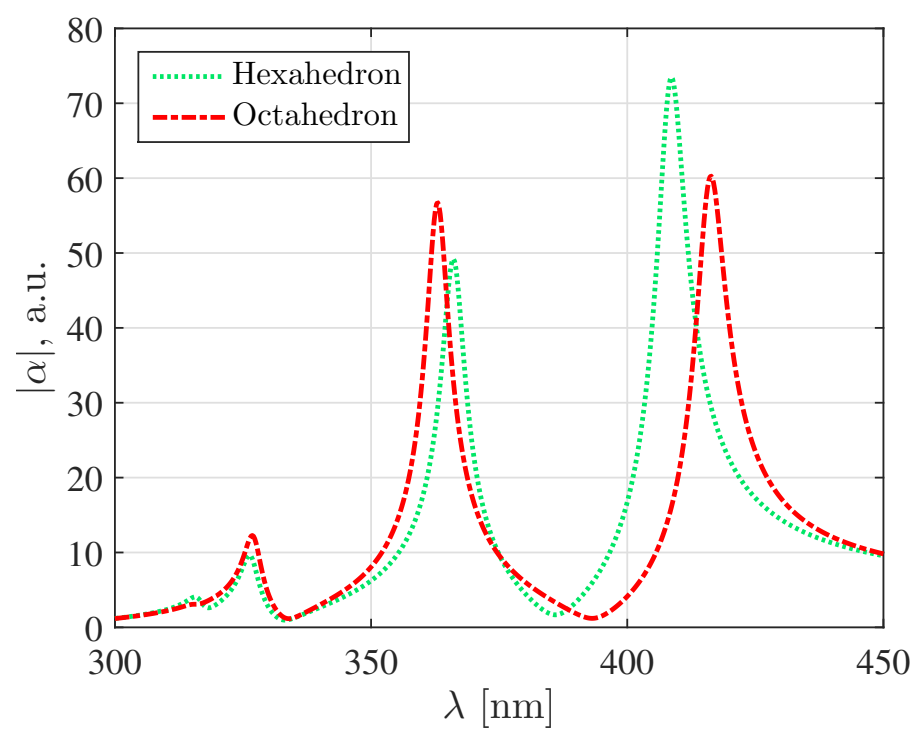

Fig. 10. Absolute value of the polarizability as a function of the wavelength (Ag Drude-model of Eq. (6)), for the hexahedral (green) and octahedral (red) nanoscatterer. Notice that octahedral solids resonate at slightly higher wavelengths than the hexahedral at their first, dipole resonance.

\section{REFERENCES}

1. X. Fan, W. Zheng, and D. J. Singh, "Light scattering and surface plasmons on small spherical particles," Light Sci. Appl. 3, e179 (2014).

2. U. Kreibig and M. Vollmer, Optical properties of metal clusters (SpringerVerlag, 1995).

3. A. Mirzaei, A. E. Miroshnichenko, I. V. Shadrivov, and Y. S. Kivshar, "Optical Metacages," Phys. Rev. Lett. 115, 215501 (2015).

4. Y. Ra'di, V. S. Asadchy, S. U. Kosulnikov, M. M. Omelyanovich, D. Morits, A. V. Osipov, C. R. Simovski, and S. A. Tretyakov, "Full light absorption in single arrays of spherical nanoparticles," ACS Photonics $p$. 150422122038008 (2015).

5. G. M. Akselrod, C. Argyropoulos, T. B. Hoang, C. Ciracì, C. Fang, J. Huang, D. R. Smith, and M. H. Mikkelsen, "Probing the mechanisms of large Purcell enhancement in plasmonic nanoantennas," Nat. Photonics 8, 835-840 (2014).

6. C. F. Bohren, "How can a particle absorb more than the light incident on it?',' Am. J. Phys. 51, 323-327 (1983).

7. H. Paul and R. Fischer, "Light absorption by a dipole," Sov. Phys. Usp. 26, 923-926 (1983).
8. A. A. Rizvi and C. H. Papas, "Power Flow Structures in Two Dimensional Electromagnetic Fields," Prog. In Electromagn. Res. 29, 261-294 (2000).

9. H. F. Schouten, T. D. Visser, and D. Lenstra, "Optical vortices near sub-wavelength structures," J. Opt. B: Quantum Semiclassical Opt. 6, S404-S409 (2004).

10. Z. B. Wang, B. S. Luk'yanchuk, M. H. Hong, Y. Lin, and T. C. Chong, "Energy flow around a small particle investigated by classical Mie theory," Phys. Rev. B 70, 035418 (2004).

11. M. Bashevoy, V. Fedotov, and N. Zheludev, "Optical whirlpool on an absorbing metallic nanoparticle." Opt. Express 13, 8372-8379 (2005).

12. M. I. Tribelsky and B. S. Luk'yanchuk, "Anomalous light scattering by small particles," Phys. Rev. Lett. 97, 1-4 (2006).

13. A. Alù and N. Engheta, "Higher-order resonant power flow inside and around superdirective plasmonic nanoparticles," J. Opt. Soc. Am. B 24, A89 (2007).

14. C. F. Bohren and D. R. Huffman, Absorption and scattering of light by small particles (John Wiley \& Sons, 2008).

15. F. Monticone, C. Argyropoulos, and a. Alù, "Layered plasmonic cloaks to tailor the optical scattering at the nanoscale." Sci. Rep. 2, 912 (2012).

16. E. Thiessen, F. X. Bronold, R. L. Heinisch, and H. Fehske, "Scattering of infrared light by dielectric core-shell particles," Phys. Rev. A 91, 043837 (2015).

17. Z. Ruan and S. Fan, "Superscattering of light from subwavelength nanostructures," Phys. Rev. Let. 105, 013901 (2010).

18. D. Gao, A. Novitsky, T. Zhang, F. C. Cheong, L. Gao, C. T. Lim, B. Luk'yanchuk, and C.-W. Qu, "Unveiling the correlation between nondiffracting tractor beam and its singularity in Poynting vector," Laser Photonics Rev. 9, 75-82 (2015).

19. P. Ylä-Oijala, M. Taskinen, and J. Sarvas, "Surface integral equation method for general composite metallic and dielectric structures with junctions," Prog. In Electromagn. Res. 52, 81-108 (2005).

20. A. H. Barr, "Superquadrics and Angle-Preserving Transformations." IEEE Comput. Graphics Appl. 1, 11-23 (1981).

21. A. Sihvola, P. Ylä-Oijala, S. Järvenpää, and J. Avelin, "Polarizabilities of platonic solids," IEEE Trans. Antennas Propag. 52, 2226-2233 (2004).

22. P. Ylä-Oijala, J. Markkanen, S. Järvenpää, and S. P. Kiminki, "Surface and Volume Integral Equation Methods for Time-Harmonic Solutions of Maxwell's Equations (Invited Paper)," Progr. In Electromagn. Res. 149, 15-44 (2014).

23. D. M. Solis, J. M. Taboada, L. Landesa, J. L. Rodriguez, and F. Obelleiro, "Squeezing Maxwell's Equations into the Nanoscale (Invited Paper)," Progr. In Electromagn. Res. 154, 35-50 (2015).

24. F. J. García de Abajo and A. Howie, "Retarded field calculation of electron energy loss in inhomogeneous dielectrics," Phys. Rev. B 65, 115418 (2002).

25. U. Hohenester and J. Krenn, "Surface plasmon resonances of single and coupled metallic nanoparticles: A boundary integral method approach," Phys. Rev. B 72, 195429 (2005).

26. A. M. Kern and O. J. Martin, "Surface integral formulation for 3D simulations of plasmonic and high permittivity nanostructures," J. Opt. Soc. Am. A 26, 732-740 (2009).

27. J. M. Taboada, J. Rivero, F. Obelleiro, M. G. Araújo, and L. Landesa, "Method-of-moments formulation for the analysis of plasmonic nanooptical antennas," J. Opt. Soc. Am. A 28, 1341-1348 (2011).

28. P. Ylä-Oijala and M. Taskinen, "Calculation of CFIE impedance matrix elements with RWG and $\mathrm{n} \times \mathrm{RWG}$ functions," IEEE Trans. Antennas Propag. 51, 1837-1846 (2003).

29. S. Järvenpää, M. Taskinen, and P. Ylä-Oijala, "Singularity subtraction technique for high-order polynomial vector basis functions on planar triangles," IEEE Trans. Antennas Propag. 54, 42-49 (2006).

30. M. T. H. Reid and S. G. Johnson, "Efficient Computation of Power, Force, and Torque in BEM Scattering Calculations," IEEE Trans. Antennas Propag. 63, 3588-3598 (2015).

31. A. Ishimaru, Wave Propagation and Scattering in Random Media, vol. 12 (John Wiley \& Sons, 1999).

32. L. Euler, "Elementa doctrinae solidorum," Novi Commentarii Academiae Scientiarum Petropolitanae 4, 109-140 (1758).

33. A. Jaklic, A. Leonardis, and F. Solina, Segmentation and recovery of 
superquadrics, vol. 20 (Springer Science \& Business Media, 2013).

34. E. Abbena, S. Salamon, and A. Gray, Modern differential geometry of curves and surfaces with Mathematica (CRC press, 2006).

35. H. Wallén, H. Kettunen, and A. Sihvola, "Composite near-field superlens design using mixing formulas and simulations," Metamaterials $\mathbf{3}$, 129-139 (2009).

36. P. B. Johnson and R.-W. Christy, "Optical constants of the noble metals," Phys. Rev. B 6, 4370 (1972).

37. U. Kreibig, "Electronic properties of small silver particles: the optical constants and their temperature dependence," J. Phys. F: Met. Phys. 4, 999-1014 (1974).

38. R. Fuchs, "Theory of the optical properties of ionic crystal cubes," Phys. Rev. B 11, 1732-1740 (1975).

39. B. Sturman, E. Podivilov, and M. Gorkunov, "Universal plasmonic properties of two-dimensional nanoparticles possessing sharp corners," Phys. Rev. B 87, 115406 (2013).
40. D. C. Tzarouchis, P. Ylä-Oijala, T. Ala-Nissila, and A. Sihvola, "Shape effects on surface plasmons in spherical, cubic, and rod-shaped silver nanoparticles," Appl. Phys. A 122, 298 (2016).

41. T. V. Raziman and O. J. F. Martin, "Polarisation charges and scattering behaviour of realistically rounded plasmonic nanostructures," Opt. Express 21, 21500 (2013).

42. V. Klimov, G. Y. Guo, and M. Pikhota, "Plasmon resonances in metal nanoparticles with sharp edges and vertices: A material independent approach," J. Phys. Chem. C 118, 13052-13058 (2014).

43. E. Shamonina, V. Kalinin, K. Ringhofer, and L. Solymar, "Short dipole as a receiver: effective aperture shapes and streamlines of the Poynting vector," IEE Proc. : Microwav. Antenn. Propag. 149, 153 (2002).

44. O. S. Vartia, P. Ylä-Oijala, J. Markkanen, S. Puupponen, A. Seppälä, A. Sihvola, and T. Ala-Nissila, "On the applicability of discrete dipole approximation for plasmonic particles," J. Quantitative Spectroscopy Radiative Transfer 169, 23-35 (2016). 